\title{
Notes sur les babesioses et l'anaplasmose des bovins à Madagascar II. Influence de la splénectomie
}

\author{
por G. UILENBERG
}

\begin{abstract}
RÉSUMÉ
L'auteur étudie l'influence de la spléneclome chez les bovins, tant sur la période d'incubation après ınoculatıon par voie sous-cutanée de sang parasıté, que sur le parasitisme chez les animaux naturellement porleurs de parasites, ainsi que sur l'évolution du parasıtısme à la suife de la transmission par la seringue de parasites d̀ des anımaux ındemnes, chaque fos tant en ce qui concerne B. bigemina et B. argentıno qu'A. marginale et A. centrale. L'auteur étudie également l'influence que peut avorr la splénectomie sur une possible augmenta. tion des formes bourgeonnantes et triples de B. bugemina.
\end{abstract}

Les méthodes de travail ont été exposées dans la première note (UILENBERG, 1968). Une grande partie des études ayant porté sur des bovins splénectomisés, nous indiquerons en résumé l'influence de la splénectomie sur les infections en question. Le nombre d'anımaux splénectomisés, d̀ l'exclusion de ceux cités dans les travaux de 1962 (RAYNAUD, 1962, 1962a, RAYNAUD et UILENBERG, 1962) est de 139. celui des animaux non splénectomisés étudiés s'élève à environ 50 au laboratore, et plus de 1.000 si on $y$ inclut les prémunitions sur le terrain.

10 Influence sur la période d'incubation, après inoculation de sang infectreux par voie sous-cutanée

(Les détails sur la période d'ıncubation après inoculation de sang par voie intraveineuse seront également donnés dans des remarques séparées.)

A. - B. Bigemina, B. Argentina et A. Marginale.

(Les résultats sur $A$. centrale sont donnés d̀ part, pour des raısons explıquées plus loın.) Voir Tableau 1.

Remorques :

\section{B. Bigemina.}

a) Aucune corrélation entre la quantité de sang ou de parasites inoculés et la durée de l'incubation n'a pu être constatée, nı sur les animaux splénectomisés, nI sur les autres, ce qui concorde avec les résultats de ROSENBUSCH et GONZALEZ (1925) et, en ce qui concerne les splénectomisés, avec les observations de KEMRON e. a. (1964). Ces derniers trouvent par contre une corrélation négative entre le nombre de parasites inoculés et la durée de l'incubation, en ce qui concerne les bovins non splénectomisés. Une telle corrélation n'a pas pu être remorquée dans nos expériences, mais les observalıons sur cette catégorie d'anımaux ne sont pos assez nombreuses pour permettre des conclusions certaınes. (A titre d'exemple: 4 animaux non splénectomısés injectés le même jour avec du sang d'un même donneur à la même dose chacun (moins de 5 millions de parasites) ont eu une pérıode d'ıncubation parasitaire de 5, 9 et 11 jours.)

b) L'incubation thermıque n'était jamaıs plus courte que l'incubation parasitare (39 observations). Dans 38 cas, totalısant les animaux 
TABLEAU $N^{d}$ I

Durée de la période d'incubation après tnoculation par voie sous cutanée de sang infectleux

\begin{tabular}{|c|c|c|c|c|c|c|c|c|c|}
\hline \multicolumn{5}{|c|}{ Incubation de l'accēs parasttaire } & \multicolumn{5}{|c|}{ Incubation de l'accēs thermique } \\
\hline & $\mathrm{N}$ & $\mathrm{M}$ & Ext & rêmes & $\mathrm{N}$ & M & Ext & têm & \\
\hline & & & \multicolumn{3}{|c|}{ B. bigemina } & & & & \\
\hline A & 47 & $6,4 \pm 0,49$ & 3 & -10 & 32 & $8,1 \pm 0,57$ & 5 & - & 12 \\
\hline B & 25 & $6,5 \pm 0,69$ & 3 & -10 & 14 & $7,3 \pm 0,75$ & 5 & - & 11 \\
\hline$A+B$ & 72 & 6.5 & 3 & -10 & 46 & 7,8 & 5 & - & 12 \\
\hline & & & \multicolumn{3}{|c|}{ B, argentina } & & & & \\
\hline A & 23 & $12,0 \pm 1,18$ & 7 & -18 & 21 & $11,3 \pm 1,28$ & 6 & - & 16 \\
\hline B & 10 & $9,3 \pm 2,75$ & 5 & -16 & 12 & $12,2 \pm 1,57$ & 7 & - & 16 \\
\hline$A+B$ & 33 & 11,2 & \multicolumn{2}{|c|}{ A. marginale } & $\begin{array}{c}33 \\
\text { nate }\end{array}$ & 11,6 & 6 & - & 16 \\
\hline A & 23 & $24,6 \pm 2,81$ & 14 & -36 & 7 & 27,1 & 21 & - & 33 \\
\hline B & 7 & $17,4 \pm 2,74$ & 13 & -22 & 2 & 20,0 & 20 & - & 20 \\
\hline$A+B$ & 30 & 22,8 & 13 & -36 & 9 & 25,6 & 20 & - & 33 \\
\hline
\end{tabular}

$\mathrm{N}=$ nombre $d$ 'observations ; $\mathrm{M}=$ moyenne et intervalle de confiance pour $\mathrm{P}<0,05$;

$A=$ animaux splënectomisés $; B=$ animaux non splénectomisếs.

Incubation parasitaire : Délai entre l'injection et le premier jour où le parasile est trouvé sur frottis mince. Incubation thermique : Délai entre l'injection ef le premier jour d'hyperthermie.

Extrêmes : La durée la plus courte et la plus longue de l'incubation observée, en jours.

Obs. : Nombre d'observations. (Le nombre est souvent moins élevé pour l'ıncubation thermique que pour l'ıncubatıon parasitaire; parfois un traitement a été fait avant que la fièvre ne se développe, plusieurs anımaux ne réagirent pas thermiquement, parfois des crises dues à d'autres parasıtes sanguins ont masqué l'hyperthermie causée par le parasite en question, etc.)

splénectomisés et les autres, l'incubation thermique était plus longue que l'incubation parasitaire, de 1 à 3 jours, en moyenne 1,9. Dans un seul cas elles étaient égales, cependant de façon douteuse : l'animal présentait une hyperthermie de $40.5{ }^{\circ} \mathrm{C}$ le jour ou les premiers parasites (rares) furent trouvés dans le sang, mais la température s'était abaissée le lendemain da $39,3^{\circ} \mathrm{C}$, et ce n'est que lorsque la parasitémie fut devenue très intense au 3 e jour de l'accès, que l'hyperthermie s'éleva de nouveau à $40^{\circ} \mathrm{C}$. Ce résultat ne correspond pas à celui de SERGENT e. a. (1924), qui signale une incubation thermique plus courte que l'incubation parasitaire dans 8 cas sur 18 , et plus longue seulement 5 fois sur 18, les deux étant égales dans les autres cas ; KEMRON e. a. (1964) et BARNETT (1965) par exemple obtiennent les mêmes résultats que nous. Il est possible que d'autres parasites sanguins, inconnus en 1964, tels les Eperythrozoo, aient perfurbé les expériences de SERGENT e. $a$,
Le fait que l'incubation thermique soit plus longue que l'incubation parasitaire est valable également pour les animaux inoculés par vole intravemeuse : 10 bovins splénectomisés ont montré des incubations parasitaires de 3 à 9 jours (en moyenne 4,9), des incubations thermiques de 6 à 12 (en moyenne 7,6 (7 observations seulement)), avec des différences entre les deux incubations de 2 jours ( 5 cas) et 3 jours ( 2 cas). Aucune observation sur animaux non splénectomisés.

\section{B. Argentino.}

a) L'incubation parasitaire trouvée (par examen de frottis minces de sang périphérique) n'a qu'une valeur restreinte pour $B$. argentina; les parasites sont habituellement si rares dans le sang, qu'ils échappent facilement à l'attention. L'incubation thermique a une valeur plus grande. Les différences observées entre les deux incubations ne signifient donc rien ici.

b) ll est difficile de juger de l'influence de la 
dose de parasites inoculés sur la durée de l'incubation, les parasites étant si rares dans le sang périphérique qu'un dénombrement comparatif semble pratiquement impossible.

c) Le fait que l'incubation (parasitaire ou thermique) après injection de sang solt en moyenne plus longue pour $B$. argentina que pour $B$. bigemina, tandis que l'inverse est vrai après transmission par la tıque B. microplus, s'explıque par les résultats de recherches effectuées en Australie (HOYTE, 1961, RIEK, 1964, 1965, 1966), qui montrent que la tıque, après s'être fixée, inocule B. argentina plusieurs jours plus tôt que $B$. bigemino.

d) Ajoutons que 9 animaux (tous splénectomisés) ont été inoculés par voie introveineuse ; l'incubation parasitare était de 6 à 14 jours (en moyenne de 8,9 ). l'ıncubation thermique ( 6 observations seulement) de 6 à 14 également (en moyenne de 10,4 jours).

\section{A. Marginale.}

a) L'incubation thermique, après inoculation par voie sous-cutanée, était toujours plus longue que l'incubation parasitaire, de 4 à 9 jours, en moyenne 6 ( 9 observations).

b) Nos chiffres montrent une différence statistiquement significative pour $P>95$ p. 100 (mals non pour $P>99$ p. 100), entre la moyenne de l'incubation parasitaire sur les animaux splénectomisés et celle sur les autres anımaux. Il ne faut pas attacher beaucoup de valeur à cette différence, puisque la durée de l'incubation semble varier, entre autres, avec la dose d'anaplasmes inoculés. Cela a déjà été démontré par ROSENBUSCH et GONZALEZ (1925) et c'est certainement vrai pour A. centrale (voir plus loin), ef pour les animaux inoculés avec $A$. marginale par voie Intraveineuse (voir remarque $c$ )). Une comparaison des résultats sur animaux inoculés par voie sous-cutanée avec des doses plus ou moins grandes d'A. marginale donne une indication dans le même sens, maıs les différences ne sont pas statistiquement significatives.

c) 10 animaux (tous splénectomısés) ont été inoculés par voie intraveineuse ; l'ıncubation parasitaire variait de 8 à 34 jours, avec une influence nette de la dose de parasites : 5 animaux inoculés avec des doses de 50 à $100 \mathrm{ml}$ de sang montrant de nombreux anaplasmes sur frottıs ont eu des incubations parasitaires de 8 à 13 jours (10.6 en moyenne) ; 5 anımaux inoculés avec des doses de 10 à $50 \mathrm{ml}$ de sang de porteurs ne montrant pas ou très peu de parasites sur frottis ont incubé de 14 à 34 jours $(14,15,17,18$ et 34). L'incubation thermique a pu être détermınée dans 4 cas; elle étalt de 6 à 9 jours plus longue que l'incubation parasitaire (en moyenne de 7,5).

\section{B. - A. Centrale.}

Une comparaison entre les animaux splénectomisés et les autres n'est pas possible sur l'ensemble des animaux, l'influence de la dose d'anaplasmes inoculés sur la pérıode d'ıncubation étant trop importante.

a) Animaux splénectomisés ınoculés avec des doses de 40 à $50 \mathrm{ml}$ de sang contenant de très nombreux $A$. centrale. L'incubation porasitaire variait de 6 à 10 jours, avec une moyenne de 8 (4 observations); l'incubation thermique étalt de 13 à 18 jours, en moyenne 15 (4 observations), de 4 à 9 jours (en moyenne 7 ) plus longue que l'incubation parasitaire. Aucun animal non splénectomisé n'a reçu de telles doses d'anaplasmes.

b) Animaux inoculés avec des doses de 5 à $20 \mathrm{ml}$ de sang contenant d'assez nombreux anaplasmes. L'incubation parasitare varialt de 17 à 22 jours (4 observations); elle était sur 3 splénectomisés de 17,18 et 20 jours et sur l'unique animal non splénectomisé de 22 jours. L'incubation thermıque n'a pu être déterminée que sur un seul animal splénectomisé ; elle était de 28 jours, de 8 jours plus longue que l'incubation parasitalre.

c) Anımaux inoculés avec du sang de porteurs chroniques, ne montrant pas ou de très rares anaplasmes sur frottıs (doses de 10 à $50 \mathrm{ml}$ ). L'incubation parasitarre variait de 21 à 36 jours, en moyenne 30 ( 8 observations) ; elle était sur les splénectomısés (4 observations) de 21 à 36 , avec une moyenne de 31 jours, sur les autres anımaux (également 4 observatıons) de 24 à 35 , en moyenne de 29 jours. Aucune différence statistiquement significative. L'incubation thermique n'o pu être détermınée que sur 2 anımaux splénectomisés; elle était de 49 et 50 jours, 13 et 14 jours plus longue que l'incubation parasitaire.

Remarque :

Ajoutons que 3 animaux ont été inoculés par vore Intraverneuse. Un anımal splénectomisé, ayant 
reçu $50 \mathrm{ml}$ de sang contenant d'assez nombreux anapiasmes a eu une incubation parasitaire de 14 jours, une incubation thermique de 22 . Un autre splénectomisé reçoit $100 \mathrm{ml}$ de sang d'un porteur chronique, et les premiers parasites ne furent observés sur frottis qu'après 50 jours ; l'incubation thermique n'a pas été déterminée. Finalement un animal non splénectomisé fut inoculé avec $100 \mathrm{ml}$ de sang contenant d'assez nombreux parasıtes; l'incubation parasitaire fut de 8 jours, l'incubation thermique n'a pas pu être déterminée.

\section{$2^{\circ}$ Influence de la splénectomie sur la parasitémie chez les animaux porteurs des parasites}

BÜCK (1933) a été le premier à Madagascar à pratıquer une splénectomie sur bovin. RAYNAUD (1962) a commencé à employer l'ablation de la rate pour la prospection systématique des hématozoaires de bovins dans le pays; la même année il donne un résumé de ses résultats (RAYNAUD, 1962a). Nos propres résultats sont les suivants :

\section{A. - B. Bigemina.}

Le parasite est observé pour la première fois de 1 à 17 jours après lasplénectomie, en moyenne 3,5 jours (46 observations); dans la grande majorité des cas il apparaît après 1 à 7 jours (44 cas), la pérıode latente étalt de 12 et 17 jours respectivement dans 2 autres cas. (A noter qu'une période de 1 jour signifle souvent qu'une faible parasitémie existait déjà au moment de la splénectomie.) (RAYNAUD, 1962a, indique des extrêmes de 1 d̀ 9 jours, avec une moyenne de 4 (30 cas) ; BÜCK (1933) trouve sur le vedu opéré par lui une période de 23 jours, donc anormalement longue.)

La rechute après l'opération est loin d'être toujours mortelle. 18 des 46 animaux n'ont pas été traités pour la première rechute, la parasitémie restant modérée, et ne sont pas morts; mais dans 2 de ces 18 cas, une rechute secondarre fut si importante qu'un traitement fut estimé nécessaire. (Ces 2 animaux étaient indemnes d'Anaplasma : une occultation parasitaire d'un accès à $B$. bigemina, temporarrement supprımé par la sortie d'A. marginale (vorr RAYNAUD, 1962a) ne peut donc pas être en cause.)
Les 28 autres ont été traités et guéris avec un piroplasmicide, mais une proportion inconnue auralt certainement guérı sans cette intervention (dans plusieurs cas un traitement au trypanbleu a été fait uniquement pour ne pas gêner l'observation d'une éventuelle sortie de B. orgentma). En général on constate que la majorité des veaux guérissent sans traitement, mais que la multiplication des parasites sur les adultes est si intense, que des symptômes de maladie apparaissent (abattement, hémoglobınurie) et qu'un traitement semble nécessaire; 11 y a des exceptions pour les deux catégories.

La parasitémie maximale de la première rechute après l'opération montre des variations d'amplitude entre faible à très élevée. Dans un cas elle était si faible qu'elle aurat facilement pu passer inaperçue: I'animal ne montrait que de très rares parasites le $17 \mathrm{e}$ jour et pendant1 jour seulement. Son sang, inoculé à un bovin splénectomısé indemne, a provoqué un accès parasitaire et thermique important, nécessitant un traitement ; Il ne semble donc pas s'agır d'un affaiblissement du parasite. Ce cas indique la possibilité qu'une certaine proportion, faible d'ailleurs, de parteurs de B. bigemina ne solt pas découverte par splénectomie.

Notons encore la rareté de réactions thermiques lors des rechutes après splénectomie, qu'il s'agisse de rechutes primaires ou secondaires.

L'évolution de la parasitémıe, après la première rechute, est également très variable. Parfols une faible parasıtémı persiste de façon pratiquement continue (dans un cas pendant toute la durée d'une observation de 6 mois), dans d'autres cas on ne retrouve plus les parasıtes après la rechute initiale, même en l'absence de traitement. Mais sur la plupart des animaux les Babesiae réapparassent de temps en temps, en nombre variable, le plus souvent faible, sans régularité ; ces apparıtıons deviennent de plus en plus rares et brèves au cours de l'observation. Dans une minorité des cas it y a des rechutes secondaires si importantes qu'elles semblent nécessiter un traitement, rechutes suivies tout comme la première si elle est importante, par des lésions sanguines d'anémie.

\section{B. - B. Argentino.}

Le parasite apparaît de 5 à 17 jours après la 
splénectomie, en moyenne 8,9 jours (7 observations); sur 5 animaux en 5 d̀ 7 jours, sur 1 après 14, et sur 1 après 17 jours. (RAYNAUD, 1962a, trouve une période latente de 4 à 7 jours, en moyenne 5,6 ( 5 cas).)

Un traitement ne semblait nécessaire que sur 2 des 7 anımaux : un adulte ayant de nombreuses B. argentino dans le sang, une température de 3909 , et des symptômes nerveux, l'autre âgé d'un an et demi, avec d'assez nombreux parasites, une température de 3903 et de l'abattement; tous les 2 guérirent après traitement. Les 5 autres bovins, âgés de 8 à 22 mols, ne montraient que de très rares à assez rares B. orgentina, un seul avalt une légère hyperthermie ( 3905$)$, et aucun ne présentalt des symptômes de maladie; tous ont survécu.

Il est certain qu'une proportion élevée de porteurs de ce parasite échappe à la détection après splénectomie. En effet, à l'opposé de ces 7 résultats positıfs, nous avons également 7 exemples où le parasite ne fut pas trouvé sur frottis de sang après l'opération, mais où il a été prouvé ultérieurement qu'ils en étalent pourtant porteurs, soit par inoculation de sang à des bovins indemnes, soit en trouvant $B$. argentına dans les capillaıres du cortex cérébral après abattage en fin d'expérience. Etant donné que de nombreux autres bovins négatıfs sur frottıs pour $B$. argentina après l'opération (mais positifs pour B. bigemina ef d'autres hématozoaires) n'ont pas été éprouvés de cette façon, il semble certaın que la proportion de porteurs révélés par splénectomie est inférieure à 50 p. 100. Cela tient sans doute, en partie tout au moins, à la rareté habıtuelle de ce parasite dans le sang périphérique. Pour avorr une meilleure idée de la proportion de porteurs dans une population donnée, Il faudrat inoculer du sang à des veaux sûrement indemnes (nés et élevés à l'abri de tıques). On peut encore utiliser la méthode de CALLOW et JOHNSTON (1963), qui trouvent que des porteurs sains montrent très souvent de rares $B$. argentina dans les capillaires du cortex cérébral. Cette méthode révèle sans doute une plus grande proportion des porteurs que la splénectomie; nous avons trouvé, en 1965 , de très rares $B$. argentina sur des frottis du cortex cérébral de 14 sur 20 zébus adultes pris au hasard à l'abattor de Tananorive (observation non publiée). Par exemple la splénectomie de 23 bovins, exposés à B. microplus, qui tous montralent après l'opération B. bigemino et $A$. marginale, ne mettait en évidence B. argentina que sur 4 sujets.

Souvent, après la première rechute consécutive d̀ la splénectomie, $B$. argentino n'est plus rencontrée sur frottis mince: parfois Il y a des rechutes secondaıres, lımitées à de très rares parasites observés pendant un ou quelques jours, sans symptômes clınıques (sauf parfois une légère hyperthermie), et sans régularité.

\section{C. - A. Marginale.}

Le parasite fait son apparition de 1 à 16 jours après l'opération, en moyenne 6,1 jours (32 observatıons) ; la pérıode latente était sur 2 anımaux seulement supérieure d̀ 10 jours, respectivement 13 et 16. (RAYNAUD, 1962a, trouve des extrêmes de 1 à 34 jours, avec une moyenne de 8,6 (36 cas), tandis que BÜCK (1933) trouve une période latente de 13 jours.)

19 des 32 anımaux n'ont pas été traıtés pendant la première rechute après l'opération; 3 sur 19 sont morts au cours de l'accès $(11,14$ et 15 jours après la splénectomie, respectıvement 10,9 et 10 jours après l'apparition des premiers anaplasmes) ; 6 autres des 19 sujets ont été abattus (pour d'autres raisons) à un stade de parasıtémie encore relativement faible, et il n'est pas possible de savorr s'ils auraient guérı spontanément ou non; les 10 autres ont guéri sans traltement, bien que la parasıtémie fût devenue importante sur certains.

13 des 32 anımaux splénectomısés ont été traités et guéris (par l'oxytétracycline ou la chlortétracycline) pendant l'accès, mals une proportion inconnue aurait certainement guérı sans cela.

La rechute est donc loın d'être toujours mortelle en l'absence de traitement; comme pour B. bigemina, le pourcentage des veaux guérissant sans traitement semble plus important que celui des adultes, sans qu'il y ait une règle fixe pour l'un ou l'autre groupe.

Lo parasitémı maximale de la première rechute peut varier de farble à très élevée, mais nous n'avons pas observé de rechute si faible qu'elle pût échapper à l'examen.

La première rechute est presque toujours suivie par une parasitémie décelable au microscope, persistant pendant longtemps, entrecoupée de temps en temps, sans régularité, de rechutes 
secondaires, parfois aussi importantes que la première (et suivies comme celle-ci d'une anémie importante), mais tout rentre dans l'ordre spontanément. Ceci va à l'encontre des résultats donnés par ROUSSELOT (1953), qui volt tous les veaux splénectomisés mourir d'accès postopératoires; y aurait-il une différence de virulence entre les souches africaines et malgaches? Les rechutes secondaires deviennent à la longue plus rares et moins importantes, et il y a parfois des périodes où le parasite n'est pas trouvé sur frottis.

Dans les cas non compliqués par des accès dus à d'autres hématozoaires, les rechutes (aussi bien la premıère que les suivantes) n'étaient jamais accompagnées d'hyperthermı.

\section{D. - A. Centrale.}

Le parasite se montre pour la première fois de 1 à 14 jours après l'opération, en moyenne après 6,3 jours (15 observations). Un seul an.mal avalt une période latente de 14 jours, les 14 autres étaient compris entre 1 et 10 jours.

Aucun des anımaux n'a été traité pendant l'accès, et tous ont guéri spontanément, bien que la parasitémie devînt parfors très élevée, et fût alors suivie d'une anémı importante.

L'intensité maxımale de la parasıtémie du premier accès est ici également très variable, et toutes les autres remarques sur $A$. marginale sont également applicables aux rechutes d'A. centrale, excepté le fait que nous ayons observé sur 3 animaux une légère hyperthermie (de $39,1^{\circ} \mathrm{C}$ à $39,4^{\circ} \mathrm{C}$ ) au cours du maximum du premier accès.

\section{$3^{\circ}$ Influence de la splénectomie \\ sur l'évolution suivant une transmission par la seringue de parasites aux animaux indemnes}

\section{A. - B. Bigemina.}

La plupart des animaux non splénectomisés indemnes, même adultes, ne meurent pas, en l'absence de traitement, après transmission par la seringue, annsi que nous avons pu l'observer lors de la prémunition artıficielle (sur laquelle des détails seront donnés dans une autre partie de ces notes).

L'infection transmise par la seringue est le plus souvent mortelie pour les bovins splénec- tomisés indemnes (veaux ou adultes), en l'absence de traltement. II y a pourtant des exceptıons. Sur 44 animaux splénectomisés inoculés, 42 ont montré une multiplication si importante des Babesioe qu'une intervention thérapeutique a été jugée nécessaire. Les animaux présentèrent en outre presque toujours de l'hyperthermie, le plus souvent voisine de $40^{\circ} \mathrm{C}$, parfois dépassant $410 \mathrm{C}$. Parmi les 42 bovins traités, 5 sont morts malgré le traitement (trop tardif).

Deux des 44 animaux n'ont pas été traités ef ont guéri spontanément; I'un (âgé de 26 mols), n'a pas montré de parasitémie importante, ni de fièvre; le nombre de parasites de l'autre (âgé de 4 mois) était élevé, mais il n'y avalt ni hyperthermie, ni hémoglobinurie. (Bien que ces guérisons spontanées soient des exceptions, ces résultats ne correspondent pas à ceux de BARNETT (1965), qui indıque que la souche de B. bigemina qu'il employait au Kenya était toujours mortelle pour les bovins splénectomisés (en l'absence de traltement), mais apathogène pour les animaux normaux; les scuches employées dans nos expériences ne peuvent certainement pas être considérées comme constamment peu pathogènes pour les bovins normaux (voir la suite de ces notes pour les détails).)

Après guérison du premier accès, l'évolution de la parasitémie sur les splénectomisés est comparable à celle observée après splénectomie de porteurs. La parasitémie est fréquemment de longue durée. Mais cela est également vrai pour les animaux normaux. Sur des veaux non splénectomisés, inoculés une seule fois avec B. bigemina, et sur lesquels aucun traitement qui pût influencer l'évolution de la parasitémie n'a été appliqué, des cas ont été rencontrés où une faible parasitémie était pratiquement contınue pendant 3 à 4 mois. Les parasites disparaissaient ensuite graduellement ou brusquement, bien qu'il ait été possible de prouver parfois (par inoculation de sang ou splénectomie) qu'ils étaient restés porteurs jusqu'au moins 6 à 7 mois après I'inoculation. De rares Babesiae ont encore été observées sur un animal 7 mois ef demı après l'inoculation. D'autres veaux, inoculés dans les mêmes circonstances, peuvent être constamment négatifs (sur frottis) à partır d'un mois après l'infection. Nous n'avons jamais observé des rechutes secondaires importantes sur des veaux normaux, non traités lors du premier 
accès : par contre, des animoux traités pour le premier accès peuvent, mais rarement, avour des rechutes secondaires importantes.

L'évolution après le premier accès sur les bovins splénectomısés, indemnes lors de l'opération, ne diffère pas de façon fondamentale de celle des animaux normaux. Le premier accès nécessitant pratiquement toujours d'être tralté, une période négative causée par le traıtement suit l'accès initial ; cette pérlode négative a une durée variable, dépendant du médıcament et de la dose employée, mais ne dépasse que rarement 2 semaines. Les parasites font ensuite leur réapparition ; Il peut alors y avoir soit une parasitémı pratiquement contınue, soit des apparitions espacées de façon irrégulıère. Une proportion importante des animaux subit des rechutes secondaires, parfois ausst importantes que le premier accès, rendant d'autres traitements nécessaires: à tıtre d'exemple : un bovın, traité lors du premier accès, retratté pour une rechute 16 jours plus tard, est mort d'une seconde rechute (non traltée) 17 jours après le deuxième traitement, avec une parasitémie très importante, de l'hémoglobınurie et une forte hyperthermie $\left(41,5^{\circ} \mathrm{C}\right.$ le jour précédant sa mort). Ces rechutes secondaires importantes sont observées dans la pérıde initiale (presque toujours pendant le premier mois), un équilibre entre les parasites et l'organisme s'installe ensuite. Dans d'autres cas, une faible parasitémie sans rechutes suit le premier accès, ou 11 y a des apparitıons irrégulières de parasites en petıt nombre. Les apparitions des Babesice se font de plus en plus rares, tout comme sur les anımaux normaux et à la longue les examens sont le plus souvent constamment négatifs, même sur les individus qui s'avèrent porteurs par Inoculation de sang à des animaux indemnes. A titre d'exemple : un bovin a pu être suivi pendant 18 mois; son sang était presque constamment positif jusqu'à 7 mols après l'inoculation, ensulte les parasites n'ont plus été observés, maıs l'inoculation de son sang, 17 mois après l'infection, prouvait qu'il étalt encore porteur ; un outre animal avait une parasıtémie presque continue pendant les 5 mols de l'observation; un trolsième présentait des parasites à intervalles irréguliers pendant les 8 mois d'observation; un autre devenait brusquement négatıf après 5 mols et ne présentait plus de parasites par la suite (observation pendant 14 mois). Nous n'avons pas observé de cas où les parasites ne réapparassent pas après le premier accès (sauf les rares cas où le traitement stérilisait l'animal de l'ınfection).

\section{B. - B. Argentina.}

Les anımaux non splénectomisés indemnes semblent très résıstants à l'inoculation par la serıngue de ce parasite (voir la partıe à venır sur la prémunition artıficielle), tout au moins avec les souches employées (et cela dépend de la race, comme nous l'avons indiqué auparavant (UILENBERG, 1968); dans de rares cas, sur des taurins, un traitement semble indiqué).

Mals les bovins splénectomisés résistent également souvent, sans que nous ayons pu observer sur cette catégorie d'animaux une influence de la race. Sur 33 animaux splénectomisés, inoculés par la serıngue, 16 n'ont pas été traıtés et aucun n'est mort à la suite de l'accès; beaucoup de ces anımaux ne réagirent que par une parasitémie et une hyperthermie (le plus souvent entre 40 et $41{ }^{\circ} \mathrm{C}$ ), quelques-uns présentalent un léger abattement et de l'inappétence pendant quelques jours. Les 17 autres ont été traités : 2 sur 17 sont morts, malgré le trattement; une proportion inconnue aurait guéri sans traitement, celul-ci étant souvent admInistré pour essayer divers médicaments et non toujours parce que l'état de l'anımal fût ınquiétant. La température maximale varıait chez 26 des 33 animaux entre $40^{\circ} \mathrm{C}$ et $41^{\circ} \mathrm{C}$, chez 6 entre $39,5^{\circ} \mathrm{C}$ et $39,9^{\circ} \mathrm{C}$, un seul animal ne présentaıt pas d'hyperthermı (malgré une parasitémie élevée). Il ne semblait pas y avoir de corrélation entre le degré d'hyperthermie, le degré de parasıtémı et les symptômes clınıques. La parasıtémie restaıt faıble dans 26 cas, dans 7 cas elle était ımportante (de façon relative les $B$. orgentına n'étant jamais sı nombreuses que les B. bigemino).

12 souches différentes ont été utilisées, obtenues soit par splénectomie de porteurs, soit par ınjection de sang d'un porteur, solt par infection par des tiques au loboratore : il ne semblait pas $y$ avar de différence marquée quant à la virulence entre les différentes souches, dont 5 ont été transmises par la serıngue 2 fols ou plus sur des anımaux splénectomısés: souvent le premier passage d'une souche « sauvage » n'exi- 
geait pas de traitement, tandis qu'un animal est mort au $5^{\mathrm{e}}$ passage d'une souche, qui n'avalt pas exigé de traitement aux $3 \mathrm{e}$ et $4 \mathrm{e}$ passages. II semble que, de façon générale, $B$. argentına injectée par la seringue ne solt pas un parasite très pathogène, ni pour les animaux normaux, nı pour les splénectomisés (à l'opposé de B. bigemina, surtout en ce qui concerne la dernière catégorle), tandis que l'infection conférée par les tıques (B. microplus) aux splénectomisés s'est toujours montrée très virulente et mortelle en l'absence de trattement (et parfois malgré le traitement).

Après le premier accès, de rares B. argentina sont observées de temps en temps sur certains animaux pendant un jour ou deux, sans symptômes cliniques (sauf parfols une légère hyperthermie), sans régularité ; sur d'autres anımaux les parasites ne réapparaissent plus, bien qu'il soit souvent possible de prouver par inoculation à d'cutres bovins qu'ils sont restés porteurs. II n'y a pas de rechutes secondaires importantes comme c'est souvent le cas pour B. bigemina.

\section{C. - A. Marginale.}

Nous n'avons pas de données relatives à la virulence des souches malgaches inoculées par la seringue aux bovins normaux adultes. Quant aux jeunes veaux normaux, ils sont très résıstants : 23 veaux de moins de 2 mors, de race taurine pure ou presque, inoculés, en souscutanée, avec $10 \mathrm{ml}$ de sang de porteurs chroniques ont tous survécu sans traltement. 19 des 23 ont pu être suivis plus ou moins régulièrement : sur 14 la parasitémie restait faible, sur 5 elle devenait assez importante, et sur 4 d'entre eux une anémıe plus ou moins importante a été observée (dont Il n'était d'ailleurs pas toujours possible de savoir si elle étalt due aux anaplasmes ou à des accès concomitants de Babesiae ou d'Eperythrozoa) ; dans aucun cas il n'y avalt des symptômes cliniques. Les 4 autres vequx, qui n'ont pas pu être contrôlés régulièrement, n'ont pas montré de maladie clınıque et n'ont pas été traités non plus.

Quant aux bovins splénectomısés, les souches malgaches isolées au hasard se sont révélées être relatıvement peu virulentes : Sur 22 anımaux splénectomısés, inoculés avec la seringue, indemnes d'anaplasmes (également d'A. centrale), et qui ont pu être suIvis régulıèrement,
16 n'ont pas été traités, et un seul est mort d'anaplasmose: sur 4 des 16 la parasitémı restait assez fable, les 12 autres avaient de nombreux ou très nombreux anaplasmes dans le sang, mais l'hyperthermie faisait souvent défaut ou, le cas échéant, dépassalt rarement $40^{\circ} \mathrm{C}$; dans 1 cas elle a attent $40,8^{\circ} \mathrm{C}$, le seul anımal qui est mort, 6 anımaux ont été traltés ; dans 3 cas le traitement semblait nécessaire étant donné la parasitémı et l'hyperthermie élevées (de $40,5^{\circ} \mathrm{C}$ à $40,9 \circ \mathrm{C}$ ), et un de ces animaux est mort d'anaplasmose le lendemain du traitement; dans les 3 autres cas le traitement (oxytétracycline ou chlortétracycline) étaıt institué en tant qu'expérience thérapeutıque au moment où la parasıtémie était encore relativement faıble.

Plusleurs souches différentes ont été utilisées, prises au hasard (obtenues après splénectomies de porteurs ou par inoculation de sang de porteurs); il ne semblait pas y avorr de différence marquée entre les souches quant à la virulence, et même les deux souches qui ont nécessité le traltement de 2 animaux se montralent peu virulentes sur d'autres.

L'évolution de la parasitémie après le premier accès est variable, mass le plus souvent il $y$ a des rechutes ( $q u$ 'il $y$ alt eu traitement ou non), souvent aussi importantes que le premier accès, avec une hyperthermie très falble ou te plus souvent nulle; ces rechutes peuvent être observées dans les quelques mois qui suivent l'infection; d̀ la longue elles deviennent moins importantes et finalement Il n'y a plus qu'une faible parasitémı continue ou alternant avec des pérıades de rémission. Aucun anımal n'est mort de rechutes, même en l'absence de traitement.

Notons encore que tous ces animaux étaient âgés de moins de 2 ans.

\section{D. - A. Centrale.}

Lo virulence de cette espèce s'est montrée être conforme à ce qui est indiqué dans d'autres pays. (La souche que nous avons, obtenue d'Israël, est toujours celle isolée en Afrique du Sud (THEILER, 1911).)

Ainsı que nos expériences de prémunition artificielle (voir une autre partie de ces notes) l'ont confirmé, elle est peu virulente pour les bovins normaux, même adultes. Environ 4.000 animaux de tous âges et de races différentes (tau- 
rins, zébus et métis) ont été inoculés avec $A$. centrale en provenance de porteurs au laboratoire, dont l'examen hématologique le jour du prélèvement confirmait la présence des parasites. Aucun animal n'a été traité, aucun symptôme clinique et aucune mortalité n'ont été observés, bien qu'ıl soit certain qu'un grand pourcentage de ces animaux, vivant dans des centres où la lutte contre les tıques était très efficace (vorr UILENBERG, 1965) n'était pas porteur d'anaplasmes.

Nous avons pu observer au laboratoire que ce parasite peut d'ailleurs parfois donner une parasitémie élevée (suivie d'anémı parfois importante), accompagnée quelquefois d'hyperthermie modérée, tout au molns sur les adultes.

Quant aux bovins splénectomisés, indemnes d'anaplasmes (y compris A. marginale).

Sur 15 anımaux inoculés par la seringue, 9 n'ont pas été traltés et ont guéri spontanément; la parasitémie restait assez faible sur 3. sur les 6 autres les anaplasmes devenaient nombreux ou très nombreux, et l'accès étalt accompagné d'hyperthermie (variant de $39,1^{\circ} \mathrm{C}$ à $40^{\circ} \mathrm{C}$ ). 6 anımaux ont été traités (oxytétracycline ou chlortétracyclıne), dans 5 cas parce que la parasitémie étalt élevée, et qu'il y avalt une fièvre de 39,5 à $40,1^{\circ} \mathrm{C}$, dans un cas au début de l'accès. II est probable que tous les anımaux auraient guéri sans traitement, mais nous ne pouvons en être certaın.

A noter que les accès dus à $A$. centrale étaient beaucoup plus souvent accompagnés d'hyperthermie que ceux dus aux souches malgaches d'A. marginale.

Les rechutes après le premıer accès sont en moyenne moins Importantes et moins nombreuses qu'avec A. morginale, et elles ne sont pas accompagnées d'hyperthermie; dans quelques cas elles étalent toutefols aussi importantes que le premier accès. Ensulte, 11 y a une faıble parasıtémie continue ou alternant avec des pérıdes de rémission.

\section{Influence de la splénectomie sur la morphologie de B. Bigemina}

RAYNAUD (1962 b) signale que la splénectomie augmenteralt la proportion des formes bourgeonnantes et triples de B. bigemina. Mals il ne se base que sur la comparason des chiffres qu'il a obtenus sur bovins splénectomisés avec ceux donnés par SERGENT e. a. (1945) sur animaux normaux; or, ces résultats sont difficılement comparables entre eux, étant donné que l'interprétation des différentes formes n'est pos identique dans les deux publications.

Nous avons comparé le pourcentage de ces formes sur quelques anımaux splénectomisés et normaux. Sur chaque anımal au moıns 200 érythrocytes infestés, pris au hasard sur toute la longueur du frottıs, ont été classés :

a) Formes bourgeonnantes = formes ayant deux petits bourgeons: ces formes ne peuvent d'aılleurs pas être séparées de façon nette des formes triples, puisqu'elles changent graduellement en formes triples, et ensuite formes géminées (doubles) ainsı que nous avons pu l'observer dans le sang frais par microscopie en contraste de phase.

b) Formes triples = les deux extensions de protoplasma sont à peu près de la même taille que la cellule mère; une séparation nette des formes bourgeonnantes d'une part et des formes géminées de l'autre n'est pas possible.

c) Formes bourgeonnantes et formes triples ensemble, les limites n'étant pas nettes de par leur transformation graduelle d'une forme à l'autre; on peut souvent hésiter sur l'une ou l'autre appellation. Pour certans animaux nous n'avons même pas distıngué les deux.

Résultots : Voir tableau II.

Ces observations montrent que la splénectomie ne paraît pas provoquer d'augmentation apparente de la proportion des formes bourgeonnantes ou triples de B. bigemına, à l'inverse de ce qu'a pu constater et écrire RAYNAUD en 1962.

Ajoutons que le pourcentage des formes doubles (deux parasites par érythrocyte, résultant de la division) variait sur ces mêmes animaux de 30,6 à 46,7 p. 100 , avec une moyenne (sur 1.707 érythrocytes infestés) de 38,1 p. 100 tandıs que le pourcentage d'érythrocytes infestés par un seul parasite (qui peut être de différentes formes, mais ne présente pas de bourgeons) étalt de 48,1 à 65.7 p. 100, avec une moyenne (sur 1.707 érythrocytes) de 57,1 p. 100 . II y avalt des différences statıstıquement sıgnificatıves entre certains animaux en ce qui concerne ces pour- 
Influence de la splénectomie sur la morphologie de $B$. bigemina.

\begin{tabular}{|c|c|c|c|c|c|}
\hline \multicolumn{6}{|c|}{ Animaux splénectomisés } \\
\hline Antmal & $\begin{array}{c}\text { Nombre } \\
\text { d'ery throcytes }\end{array}$ & $\begin{array}{c}\text { Formes } \\
\text { bourgeonnantes }\end{array}$ & & $\begin{array}{l}\text { Formes } \\
\text { triples }\end{array}$ & $\begin{array}{l}\text { Bourgeonnantes } \\
\text { plus triples }\end{array}$ \\
\hline В 73 & 210 & $4(1,9 \mathrm{p} \cdot 100)$ & 7 & $(3,3 \mathrm{p} \cdot 100)$ & $11(5,2 \mathrm{p} \cdot 100)$ \\
\hline B 85 & 215 & $5(2,3 \quad \cdots)$ & 3 & $(1,4 \quad ")$ & $B(3,7$ \\
\hline Total & $\overline{425}$ & $\overline{9}(2,1 \quad ")$ & $\overline{10}$ & $(2,4 \quad ")$ & \\
\hline B 28 & 200 & Pas fait & & Pas fait & $10(5,0 \mathrm{p} \cdot 100)$ \\
\hline В 34 & 200 & Pas fait & & Pas falt & $11<5,5$ \\
\hline B 45 & 222 & Pas fait & & Pas fait & $11(5,0$ \\
\hline Total & 1047 & - & & - & $51(4,9$ \\
\hline \multicolumn{6}{|c|}{ Animaux non splênectomisês } \\
\hline v 4 & 215 & $9(4,2$ p. 100$)$ & 5 & $(2,3$ p. 100$\rangle$ & $14(6,5 \mathrm{p} \cdot 100)$ \\
\hline 1229 & 200 & $2(1,0 \quad ")$ & 7 & $\langle 3,5$ & $9(4,5$ \\
\hline "Bleike" & 245 & $3(1,2 \quad ")$ & $\underline{6}$ & $(2,4$ & $\underline{9}(3,7$ \\
\hline Tota 1 & 660 & $14(2,1 ")$ & 18 & $(2,7$ & $32(4,8$ \\
\hline
\end{tabular}

B 73 = Accès après inoculation de sang infecté,

B 85 = Porteur; accès après splënectomie,

B 28 = Porteur; accès après splënectomie,

B 34 = Porteur ; accès après splénectomie,

B 45 = Porteur; accès après splénectomie.
V 4 = Accès après inoculation de sang infecté, $1229=$ Accès après infection par 3 . microplus,

"Bleike" = Accés aprōs inoculation de sang infecté. centages, mais cela n'avait aucun rapport avec la splénectomie, et se rapporte peut-être au stade d'évolution de l'accès.

\section{CONCLUSIONS ET RÉSUMÉ}

Des détails sont donnés sur la durée de l'ıncubation parasitaire et thermıque après inoculation par la seringue de $B$. bigemina, $B$, argentina, A. marginale et $A$. centrale, sur la durée de la période de latence postopératoire après splénectomie, sur l'évolution de la parası́emı après splénectomie et après inoculation par la seringue de ces parasites.

Aucune différence statistıquement significative n'a été trouvée entre animaux normaux et splénectomisés quant à la durée de l'incubation parasitare et thermique après ınjection de sang avec B. bigemina et B. argentina. II n'y a probablement pas non plus de différence en ce qui concerne $A$. marginale et $A$. centrale, mais l'influence de la dose d'anaplasmes injectée sur la durée de l'incubation domine le tableau.

La splénectomie de porteurs de B. bigemina,
A. marginale et $A$. centrale met toujours, ou pratiquement toujours, ces parasites en évidence ; 1 l n'en est pas de même pour B. argentina, la proportion des porteurs révélés étant probablement inférieure à 50 p. 100 . Un pourcentage important des animaux guérit spontanément des accès postopératoires de $B$. bigemina, $B$. argentina et $A$. marginale, tandis que tous les porteurs d'A. centrale guérissent sans traltement.

L'infection à $B$. bigemina transmise par la serıngue aux splénectomısés indemnes est le plus souvent mortelle en l'absence de traitement, tandis qu'un pourcentage important de splénectomisés résiste à l'ınoculation par la seringue de $B$, argentina, $A$. marginale ef $A$. centrale.

Aucune influence de la splénectomie sur le pourcentage des formes bourgeonnantes et triples de B. bigemina n'a pu être mise en évidence, à l'opposé de ce qu'écrit RAYNAUD (1962 b).

Service d'Entomologie et Protozoologie, Laboratoire Central de l'Elevage, Tananarive. Institut d'Elevage et de Médecine Vétérinaire des Pays Tropicaux. 


\section{CONCLUSIONS AND SUMMARY}

Notes on bovine babesiosis and anaplasmosis in Madagascar.

II. Influence of splenectomy

Details are given on the lenght of the parasitic and thermal incubation period after injection by the syringe with B. bigemina, B. argentına, A. morginale and $A$. centrole, as well as on the length of the Iatent periad after splenectomy, on the evolution of parasitaemia afler splenectomy and afler injection by the syringe with these parasites.

No statistically significant differences were found between normal and splenectomised animals as to the length of the parasitic and thermal incubation period after injection of blood with $B$ bigemina and $B$ argentino. There are probably no differences either as far as $A$. morginale and $A$. centrole are concerned, but the influence of the dose of anaplasms injected an the length of the incubation period dominates the picture.

Splenectomy of carriers of B. bigemino, A. morginale and A. centrale reveals these parasites always or practically always; the same is not true for $B$ argenfina, probably less than 50 p. 100 of carriers being revealed. An important percentage of the animals recover spontaneously from post-splenectomy attacks of $B$. bigemina, B. argentına and A. marginale, while all carriers of A. centrale recovered without treatment.

The infection with B. bigemina transmisted by the syringe to splenectomised cattle free of the parasite is most often fatal without treatment, while an important percentage of splenectomised anımals resist injection by the syringe with B. argentina, A morginale and A. centrale.

No influence of splenectomy on the percentage of budding and triple forms of $B$. bigemina could be shown, contrary to the opinion of Raynaud (1962 b).

\section{RESUME N}

Notas sobre las babesiosis y la anaplasmosis de los bovinos en Madagascar. II. Influencia de la esplenectomıa

El autor estudia la influencia de la esplenectomia en los bovinos sobre el periodo de incubación después de la inoculación por via subcutánea de sangre parasitada y sabre el parasıtısmo en los anımales naluralmente portadores de parásitos ası como sobre la evolución del parasitismo luego de la rransmisión por la jeringa de parásitos a anımales indemnes. Cada vez se trata de $B$. bigemina, B. argentino. A. marginale y A. centrale El autor estudia tambien la influencıa que puede tener la esplenectomia sobre un aumento posible de las formas germinantes $y$ triples de B bigemina.

\section{BIBLJOGRAPHIE}

1. BARNETT (S. F.). - The chemotherapy of Babesia bigemina infection in cattle. Res. vet. Sc. 1965, $6: 397-415$.

2. BUCK (G.). - Prémunition des zébus malgaches vis-d̀-vis des piroplasmoses et splénectomie chez un veau zébu. Bull. Soc. Path. exot., 1933, $26: 919-922$.

3. CALLOW (L. L.) et JOHNSTON (L.A.Y.).Babesia spp. in the brains of clinically normal cattle and their detection by a brain smear technique. Aust. vet. J., 1963, $39: 25-31$.

4. HOYTE (H. M. D.), - Initial development of infections with Babesia bigemina. J. Prot., 1961, $8: 462-466$.

5. KEMRON (A.), HADANI (A.), EGYED (M.), PIPANO (E.) et NEUMAN (M.). - Studies on bovine piroplasmosis caused by Babesia bigemino. Ill. The relationship between the 
number of parasites in the inoculum and the severity of the response. Refuah. Vet., 1964, $21: 112-108$.

6. RAYNAUD (J.P.). - Prospection des hématozoaires et tiques de bovins à Madagascar. l. Recherches dans la province de Tananarive. Rev. Elev. Méd. Vét. Poys Trop., 1962, 15 : 137-145.

7. RAYNAUD (J. P.). - Splénectomie des bovins et parasites sanguins. Ann. Parasit. hum. comp., 1962a, 37 : 755-766.

8. RAYNAUD (J. P.). - Morphologie, chimiosensibilité ef réactions immunitaires de souches de Babesia bigemina (Smith et Kilborne, 1893) mises en évidence par splénectomie de [bovins. Rev. Elev. Méd. Vét. Pays Trop., 1962b, 15 : 167-179.

9. RAYNAUD (J.P.) et UILENBERG (G.). Prospection des hématozoaires et tiques de bovins à Madagascar. II. Recherches complémentaires et conclusions. Rev. Elev. Méd. Vét. Poys Trop., 1962, $15:$ 147-153.

10. RIEK (R. F.). - The life cycle of Babesia bigemina (Smith \& Kilborne, 1893) in the tick vector Boophilus microplus (Canestrini). Aust. J. agric. Res., 1964, 15 : 802-821.

11. RIEK (R.F.). - The cattle tick and tick fever. Aust. vet. J., 1965, 41 : 211-216.

12. RIEK (R. F.). - The life cycle of Babesia argentino (Lignières, 1903) (Sporozoa : Piroplasmidae) in the tick vector Boophilus microplus (Canestrini). Aust. J. agric. Res., 1966, $17: 247-254$.

13. ROSENBUSCH (F.) et GONZALEZ (R.). Beitrag zum Studium der Tristeza. I. Mitteilung. Arch. Protistenk., 1925, 50 : 443-485.

14. ROUSSELOT (R.). - Notes de parasitologie tropicale. Tome I. Parasites du sang des animaux. Paris, Vigot Frères, 1953 (152 p.).

15. SERGENT (E.), DONATIEN (A.), PARROT (L.) et LESTOQUARD ( $\left.F_{1}\right)$. - Etudes sur les piroplasmoses bovines. Institut Pasteur d'Algérie, 1945 (816 p.).

16. SERGENT (E.), DONATIEN (A.), PARROT (L.), LESTOQUARD (F.), PLANTUREUX (E.) et ROUGEBIEF (H.). - Les piroplasmoses bovines d'Algérie. Arch. Inst. Pasteur. Algér., 1924, 2 : 1-146.

17. THEILER (A.). - Further investigations into anaplasmosis of South African cattle. Ist. Rept. Director Vet. Res., South Africo, 1911 : 7-46.

18. UILENBERG (G.). - Influence du détiquage sur la présence de parasites sanguins chez les bovins malgaches observés après splénectomie. Indications pratiques pour la lutte contre les hématozoaires pathogènes. Rev. Elev. Méd. Vét. Pays Trop., 1965, 18 : 165-173.

19. UILENBERG (G.). - Notes sur les babésioses ef l'anaplasmose des bovins à Madagascar. I. Introduction. Transmission. Rev. Elev. Méd. Vét. Poys Trop., 1968, 21 . 4 : 467-74. 